\section{$\mathbf{m} / \mathbf{S}$}

médecine/sciences 1993; 9: 1182-3

\title{
NOUVELLES PERSPECTIVES OSSEUSES
}

\section{Francis H. Glorieux}

\section{ADRESSE}

F.H. Glorieux : docteur en médecine, docteur ès sciences. Hôpital Shriners, unité de génétique, 1529, avenue Cedan, Montréal, Qué- bec, H3G 1A6 Canada.

\section{TIRÉS A PART}

\section{F.H. Glorieux.}

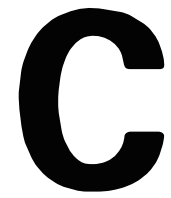

omme dans d'autres disciplines de la médecine, la compréhension de la physiopathologie des nombreuses conditions affectant le développement et l'intégrité du système osseux a été profondément améliorée par le développement explosif des techniques de biologie cellulaire et moléculaire. Cette évolution, amorcée par la découverte de la nature hormonale de la vitamine $\mathrm{D}$, a progressivement fait dériver l'étude et le traitement des maladies osseuses des domaines de la néphrologie et de l'endocrinologie classique vers ceux de la génétique, de l'oncologie, de l'endocrinologie moléculaire et de la pharmacologie. Si les concepts de base demeurent inchangés, les nouvelles techniques permettent maintenant d'en disséquer les mécanismes, ce qui aboutira, à plus long terme, à maîtriser la physiopathologie et le traitement des ostéopathies congénitales et acquises.

L'étude du développement et de la physiologie du tissu osseux était traditionnellement orientée dans deux directions liées aux rôles fondamentaux du squelette dans l'organisme. D'une part, son rôle mécanique de support et de protection des autres organes du corps, et de l'autre, son rôle endocrinien de réservoir de minéraux permettant, grâce à l'action concertée de la vitamine $\mathrm{D}$, de la parathormone et, peut-être, de la calcitonine, de maintenir la concentration extracellulaire du calcium dans des limites très étroites, un élément critique pour de nombreuses fonctions biologiques. Cette dualité de fonction se reflète dans la structure osseuse. L'os compact ou cortical qui se retrouve principalement dans la diaphyse des os longs et dans les tables des os plats, assure la résistance du squelette aux forces physiques qui s'y appliquent. L'os spongieux ou trabéculaire, en revanche, fournit une très large surface d'échange avec l'espace extracellulaire et peut donc être considéré comme l'os endocrinien.

De plus, il enchâsse le stroma médullaire, incluant les précurseurs des ostéoblastes, et les éléments hématopoiétiques comprenant les précurseurs des ostéoclastes.

Le tissu osseux est en constant renouvellement par l'action concertée des ostéoclastes et des ostéoblastes qui, respectivement, résorbent et produisent la matrice osseuse. L'équilibre entre ces deux actions régit la croissance, la masse osseuse et les échanges minéraux. Pendant longtemps, le contrôle de cet équilibre a été dévolu, d'une part, aux effets mécaniques de la contraction musculaire sur le périoste et, d'autre part, aux hormones contrôlant l'homéostase phosphocalcique. Ces éléments n'expliquent cependant ni la régulation du développement et de la croissance de l'os, ni des maladies telles que l'ostéoporose postménopausique, la maladie de Paget, les pseudarthroses ou le myélome multiple. Dans un premier volet, la découverte du rôle possible de certaines familles de proto-oncogènes dans le contrôle du développement du squelette ouvre des perspectives qui sont discutées par R. SaintArnaud dans ce numéro de médecine/sciences ( $p .1243)$. D'autre part, l'expansion rapide de nos connaissances de l'implication des cytokines dans de nombreuses fonctions organiques et cellulaires n'a pas épargné le métabolisme osseux. Il est maintenant reconnu que l'activité des cellules osseuses est influencée par plusieurs cytokines, soit isolément, soit en association. Celles-ci comprennent les interleukines (IL), les interférons (IFN), les facteurs de croissance (GF) et les facteurs stimulant les colonies cellulaires (CSF). 
Plusieurs d'entre elles font aussi partie de "superfamilles" de peptides. Ainsi TGFß, une molécule présente aux sites de formation osseuse (incluant la guérison de fractures) fait partie de la même famille que les protéines morphogéniques de l'os (BMP), un groupe de facteurs ostéoinducteurs, extraits de la matrice osseuse. Par ailleurs, la plupart des cytokines agissant sur l'os sont sous contrôle partiel des hormones classiques; c'est ainsi que la parathormone paraît stimuler la production d'IL1, les œestrogènes celle d'IGF-I et la vitamine $\mathrm{D}$ celle de TGF $\beta$.

L'importance probable de ces facteurs dans la physiopathologie de certaines maladies est soulignée par le fait qu'un défaut de synthèse de M-CSF produit une forme d'ostéopétrose (défaut de résorption osseuse) chez la souris. La suppression du phénotype par injection de M-CSF démontre le rôle de ce facteur dans la différenciation des ostéoclastes. L'observation qu'une ostéopétrose est également induite par l'inactivation de l'expression des protooncogènes $c$-fos et s-src souligne l'hétérogénéité d'affections pourtant cliniquement similaires.

D'autres maladies commencent à être reliées spécifiquement à certaines cytokines. C'est ainsi que TNF $\beta$ est, croit-on, le facteur clé de résorption dans le myélome multiple, que
l'IL6 pourrait être impliquée dans la maladie de Paget, et que l'ILl et les TNF $\alpha$ et $\beta$ sont probablement des intermédiaires importants de l'augmentation de résorption osseuse qui accompagne certains états inflammatoires. Si l'on considère spécifiquement l'ostéoporose post-ménopausique, la maladie vedette de par ses implications médicales et sociales, il est maintenant établi que la production d'IL1, de TNF $\beta$, de GMCSF et d'IL6 est augmentée après ovariectomie, suggérant que l'un ou plusieurs de ces facteurs puisse être important dans l'induction de la perte osseuse qui suit la ménopause. Plusieurs questions restent à résoudre pour déterminer la hiérarchie d'action des diverses cytokines impliquées dans le métabolisme osseux. Quels sont, par exemple, les facteurs importants dans la physiologie normale de l'os, par contraste avec ceux qui sous-tendent un processus pathologique? Y a-t-il d'autres facteurs présents dans la matrice osseuse qui jouent un rôle (le TGFß inclus dans la matrice pourrait être un facteur clé dans le couplage entre formation et résorption osseuse) ? Pourquoi y a-t-il autant de différentes cytokines et comment s'associent-elles ou s'opposent-elles pour induire des modifications métaboliques au niveau du squelette? Enfin y a-t-il un polymor- phisme génétique? Un type familial d'ostéoporose précoce semble associé à des formes alléliques particulières du récepteur de la vitamine $\mathrm{D}$. Y aurait-il aussi une prédilection individuelle à développer des maladies telles que l'ostéoporose qui ait son origine dans l'activation ou la neutralisation de cytokines spécifiques?

Un autre volet tout aussi passionnant est centré sur l'utilisation des cytokines comme médicaments. Cela concerne non seulement l'ostéoporose et les hypercalcémies mais aussi des problèmes "locaux » tels que la non-guérison de fractures, les pertes de substance osseuse (traumatiques, post-chirurgicales ou par défaut de développement) ou la fixation des prothèses articulaires par ostéointégration (croissance osseuse à l'intérieur même de la prothèse). Les problèmes pharmacologiques à résoudre sont importants. Les questions de doses, de toxicité et de disponibilité du facteur actif à l'endroit voulu sont des points critiques. Une fois résolus, il n'y a aucun doute qu'une nouvelle ère sera ouverte où non seulement nous comprendrons mieux les mécanismes intimes de nombreuses affections osseuses non encore maîtrisées, mais où nous disposerons aussi d'agents thérapeutiques spécifiques pour les contrôler 